\title{
A interferência do módulo de resiliência do concreto asfáltico no período de projeto de pavimentos rodoviários
}

\section{Erico Aliaga Cavaleiro' ${ }^{1}$, Francisco Heber Lacerda de Oliveira ${ }^{2}$}

${ }^{1}$ Instituto Federal de São Paulo, ericoaliaga@gmail.com

2Universidade Federal do Ceará, heber@det.ufc.br

\section{Recebido:}

21 de agosto de 2017

Aceito para publicação:

12 de dezembro de 2017

Publicado:

30 de abril de 2018

Editor de área:

Francisco Thiago Aragão

\section{Palavras-chaves:}

Dimensionamento,

Vida Útil,

Módulo de Resiliência,

Desempenho.

\section{Keywords:}

Pavement Design,

Design Life,

Resilience Module,

Performance.

DOI:10.14295/transportes.v26i1.1456

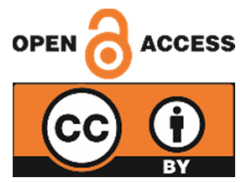

\begin{abstract}
RESUMO
Os pavimentos rodoviários são dimensionados para atender uma determinada vida útil em função dos materiais e do tráfego. A análise mecanicista prevê que o Módulo de Resiliência do Concreto Asfáltico será constante ao longo de sua vida útil, fato divergente ao encontrado na literatura, ao envelhecimento natural do ligante e às condições climáticas. Neste artigo foi possível constatar, por meio de uma verificação teórico-mecanicista, que pavimentos asfálticos dimensionados para tráfegos moderado, pesado ou muito pesado, independente da capacidade de suporte do subleito, podem perder até $78 \%$ da sua vida útil a partir do terceiro ano de operação se o Módulo de Resiliência do Concreto Asfáltico obedecer a uma tendência decrescente, e um ganho de até $172 \%$ para uma tendência crescente. Desse modo, constata-se a importância de se desenvolver modelos de desempenho que considerem os fatores que interferem no comportamento do Concreto Asfáltico para que se possa elaborar projetos mais racionais.
\end{abstract}

\begin{abstract}
Road pavements are planned to meet certain design life depending on the materials and the traffic. The mechanistic analysis predicts that the Asphalt Concrete Resilience Module will be constant along its design life, a fact that diverges from the written material available found on the subject, the natural aging of the binder and the climatic conditions. In this paper, it was possible to verify that asphaltic pavements designed to attend moderate, heavy or very-heavy traffic, regardless of the support capacity of subgrade, can lose up to $78 \%$ of their design life from the third year of operation on, if the Asphalt Concrete Resilience Module obeys a decreasing trend, and a gain of up to $172 \%$ for an increasing trend. Thus, it is important to develop performance models that consider the factors that interfere with the behavior of Asphalt Concrete in order to develop projects that are more rational.
\end{abstract}

\section{INTRODUÇÃO}

Uma das principais preocupações na fase de projeto de uma infraestrutura rodoviária é quanto à sua vida útil. Um pavimento é projetado para atender a um determinado intervalo de tempo, chamado de período de projeto ou vida útil. Esse período, geralmente, é de 10 anos para pavimentos em concreto asfáltico e 20 anos para os de concreto de cimento Portland, podendo ser prorrogado a partir de sucessivas intervenções ao longo de sua operação. (DNIT, 2006)

De modo mais efetivo, recomenda-se que o projeto de um pavimento flexível rodoviário seja elaborado pelo dimensionamento empírico, seguido da verificação mecanicista, comparando-se valores atuantes calculados em um software e valores máximos admissíveis calculados por equações de desempenho (DER/SP, 2006). Contudo, essa verificação é feita como se o Módulo de Resiliência (MR) do Concreto 
Asfáltico Usinado à Quente (CAUQ) fosse constante ao longo de todo seu período de projeto, premissa não conforme com o que será discutido neste artigo.

São apresentadas ao longo deste artigo estudos em que foram identificadas variações crescentes e decrescentes do MR do CAUQ e suas relações com variações de temperatura, envelhecimento por oxidação, intensidade do tráfego, condições ambientais, dentre outras, impactando diretamente no período de projeto do pavimento. A importância de se compreender os fatores intervenientes nas variações do MR do CAUQ tem incentivado a elaboração de modelos mais eficientes de previsão de desempenho de pavimentos.

Assim, esta pesquisa objetiva descrever uma verificação teórico-mecanicista da variação do MR do CAUQ ao longo do período de projeto de um pavimento rodoviário, a partir do dimensionamento empírico do Departamento Nacional de Infraestrutura de Transporte (DNIT), para diferentes volumes de tráfego, tipos de materiais e de capacidade de suporte do subleito.

\section{REVISÃO DA LITERATURA}

As camadas da estrutura de um pavimento rodoviário podem ser constituídas por diversos materiais. A escolha desses ocorre em função do tipo de estrutura a ser implantada, das características do perfil geotécnico da região, do tráfego que solicitará a estrutura e, principalmente, dos materiais disponíveis. Para a elaboração racional de um projeto de pavimento, é necessário compreender o seu princípio de funcionamento, ou seja, a mecânica do pavimento, que, por sua vez, está intimamente relacionada com duas principais variáveis: o tráfego e as características geotécnicas.

A modelagem do tráfego a que o pavimento estará sujeito durante seu período de projeto, segundo Senço (1997), é feita, no Brasil, em termos de repetição de um eixo simples de rodas duplas de 8,2 tf, que converte a passagem de diferentes eixos, com diferentes cargas e frequências de repetição, em um único valor de eixo-padrão, conhecido como Número $\mathrm{N}$.

Já as características geotécnicas são expressas pelo valor da capacidade de suporte do solo, determinado pelo Índice de Suporte Califórnia - ISC (ou California Bearing Ratio - CBR) e expansibilidade. Ele avalia, de forma indireta, a resistência ao cisalhamento dos solos e materiais granulares, comparando-a a de uma brita considerada como padrão, cuja resistência é dada pelo atrito entre os grãos. 0 ensaio é útil para avaliar o subleito e camadas superiores que contenham materiais passantes na peneira de abertura igual a $19 \mathrm{~mm}$ (DNER, 1994).

No Brasil, o Método do DNER (antigo Departamento Nacional de Estradas de Rodagem, atual DNIT) é o método oficial de dimensionamento de pavimentos flexíveis em rodovias federais (e em muitas estaduais), e se baseia nas premissas anteriores. Todavia, é sabido que os métodos existentes são concebidos de duas maneiras distintas, sendo um baseado no desempenho do pavimento (modelos empíricos), e outro a partir de teoria elástica (modelos racionais). Além disso, outras formas de projeto associam os modelos empíricos com racionais.

Nos Estados Unidos, de acordo com Bernucci et al. (2008), o CBR foi sendo progressivamente substituído pelo MR, tendo sido adotado definitivamente em 1986 para dimensionamento de pavimentos asfálticos pelo guia de projeto norte-americano da American Association of State Highway and Transportation Officials - AASHTO.

Segundo Severi et al. (1998), o dimensionamento de pavimentos asfálticos passou por algumas transformações nos últimos anos. Alguns parâmetros foram atualizados e outros concebidos, devido ao colapso de algumas estruturas, ocasionado por diversas causas, dentre as quais destacam-se: (i) o fenômeno de fadiga, responsável pelo trincamento de revestimentos asfálticos e de bases cimentadas, e (ii) as deformações plásticas provocadas por tensões de deformações cisalhantes que ocorrem em camadas granulares e no subleito.

Ainda conforme Severi et al. (1998), a capacidade estrutural pode ser entendida pelo modo que a carga aplicada pelo tráfego distribui as tensões e deformações nas camadas do pavimento, e a compatibilidade dos materiais e suas características resistentes. A análise dessa capacidade é realizada por meio 
de softwares que permitem observar as tensões existentes em todas as camadas do pavimento, sejam elas, de tração, compressão ou cisalhantes.

A aplicação dos métodos de dimensionamento mecanicistas exige o conhecimento de parâmetros elásticos dos materiais que compõe as camadas dos pavimentos, tais como o Coeficiente de Poisson e o Módulo de Elasticidade. O Coeficiente de Poisson é a relação entre as deformações transversal (perpendicular à carga) e longitudinal (na direção da carga) de um corpo sob a ação de uma carga. Por sua vez, o Módulo de Elasticidade é a relação entre a tensão e a deformação (ou a tangente inicial de uma curva tensão versus deformação) na região de deformação elástica, na qual a Lei de Hooke é válida. Esse módulo descreve matematicamente a rigidez de um material e a sua capacidade de se deformar elasticamente (recuperável), quando está sob a ação de uma carga, segundo Beer e Johnston Junior (1995).

Estudar o pavimento sob o enfoque mecanicista significa, na visão de Balbo (2007), compreender como a ação das cargas oriundas do tráfego, associadas àquelas oriundas das ações do clima e às propriedades dos materiais, promovem o aparecimento de tensões, deformações e deslocamentos. Entre os modelos matemáticos utilizados para descrever mecanicamente o comportamento dos pavimentos, pode-se citar o Método dos Elementos Finitos (MEF) e a Teoria dos Sistemas de Camadas Elásticas (TSCE).

Balbo (2007) explica que o Cimento Asfáltico de Petróleo (CAP) utilizado na produção do CAUQ o revestimento mais comumente utilizado no Brasil em pavimentos rodoviários, é considerado um material termoviscoelastoplástico. Devido à sua suscetibilidade térmica, pode sofrer alterações com o intemperismo, o que o faz perder suas propriedades iniciais. Além disso, pode se alterar também quando exposto à radiação solar, águas ácidas ou sulfatadas, graxas e óleos num processo denominado oxidação do ligante asfáltico.

Para Bernucci et al. (2008), a aplicação desse material na pavimentação pode ter seu desempenho mecânico alterado conforme a velocidade, intensidade de carregamento do tráfego e temperatura de serviço. Essa deterioração ocorrida ao longo do tempo ou de modo precoce pode ser denominada de envelhecimento.

Existem dois tipos de envelhecimento, segundo Magalhães (2004). 0 envelhecimento de curto prazo, que acontece no período de estocagem, usinagem e aplicação da mistura asfáltica e está diretamente relacionado com a temperatura e com o grau de exposição do ligante ao oxigênio. A grande superfície específica durante a usinagem associada à temperatura elevada, expõe o ligante ao oxigênio propiciando um envelhecimento acelerado. Já durante a vida de serviço do pavimento, ocorre o envelhecimento de longo prazo, que, por sua vez, está associado diretamente com o teor de vazios da mistura, temperatura, espessura da película e posição em profundidade na camada. Neste caso, é considerado um processo de envelhecimento lento.

Magalhães (2004) ainda apresentou resultados obtidos numa simulação do efeito da espessura da película no envelhecimento de curto e de longo prazo do ligante. Percebeu-se que o $\mathrm{MR}$ à $25^{\circ} \mathrm{C}(\mathrm{MPa})$, a penetração à $25^{\circ} \mathrm{C}$ e a viscosidade à $60^{\circ} \mathrm{C}$ variam menos em relação aos valores originais conforme aumenta-se a espessura da película.

Por fim, Magalhães (2004) verificou que o ligante convencional (tipo CAP 50/60) e o Asfalto Modificado por Polímero (ligante modificado, tipo AMP SBS) praticamente dobraram os valores de MR após envelhecimento de curto prazo, enquanto que o CAP 20 e o ligante modificado (tipo AMP EVA) tiveram um aumento em torno de 30\%. 0 aumento relativo do MR da mistura com Resíduo Asfáltico de Penetração (RASF) foi o menor de todos com apenas 12\% de envelhecimento de curto prazo. Este fato deve estar ligado ao baixo teor de frações voláteis no ligante RASF em comparação com os demais.

Rohde (2007), por sua vez, apresentou resultados obtidos numa simulação dos efeitos da temperatura no MR de diversos tipos de ligantes asfálticos. 0 ensaio utilizado foi o de Estufa de Filme Fino Rotativo (ou RTFOT - Rolling Thin Film Oven Test), no qual se observou a interferência da temperatura no MR dos diversos ligantes utilizados. Os resultados mostraram que, de maneira geral, quanto maior a temperatura, menor é o MR e a resistência à tração (RT). 
Levando-se em consideração essas pesquisas, foi possível constatar a interferência causada no MR do CAUQ por fatores como: (i) Espessura da camada: quanto maior a espessura, menor a superfície específica, menor a oxidação, e consequentemente, menor variação de MR (sofrem menos enrijecimento ao longo do tempo); (ii) Tipo de ligante: a natureza do CAP pode determinar variações mais significativas de MR (sofrem mais ou menos enrijecimento ao longo do tempo); (iii) Intemperismo (temperatura e radiação solar): quanto maior a temperatura menor é o $\mathrm{MR}$, bem como quanto maior a exposição à radiação solar, maior é o enrijecimento ao longo do tempo, e (iv) Usinagem, estocagem e transporte: influenciam sempre no sentido de aumentar o MR devido a oxidação do ligante.

Diante desses fatores, para um dimensionamento adequado, Balbo (2007) recomenda realizar a verificação mecanicista, sendo necessário localizar os pontos de tensões, deformações e deflexões atuantes na estrutura. Na análise do pavimento como um sistema de diferentes camadas, existem posições críticas para essa verificação, que são as apresentadas na Tabela 1.

Tabela 1: Posições críticas de análise na estrutura de pavimentos flexíveis ou semirrígidos. Fonte: Balbo (2007).

\begin{tabular}{lll}
\hline Posição & Resposta & Emprego Típicos da Resposta \\
\hline Revestimento (superfície) & Deflexão & Projetos de reforço \\
Revestimento (fundo) & Deformação horizontal & Análise de fadiga \\
Camadas tratadas (fundo) & Tensão horizontal & Análise de fadiga \\
Meia altura de camadas & Deformação vertical de compressão & Análise de deformação plástica \\
Topo do subleito & Deformação vertical de compressão & Análise de deformação plástica \\
\hline
\end{tabular}

\section{MÉTODO DE OBTENÇÃO DOS DADOS}

De modo a se compreender os fatores intervenientes nas variações do MR do CAUQ foram dimensionadas 48 estruturas de pavimentos asfálticos pelo método empírico do DNIT para um período de projeto de 10 anos, diferentes volumes de tráfego e de CBR do subleito. Após o dimensionamento empírico, foi feita a verificação mecanicista das estruturas, a partir das hipóteses de MR crescentes, muito crescentes, decrescentes e muito decrescentes, e dos modelos de desempenho do CAUQ previstos em DNIT (2006), comparando a interferência dessas hipóteses no período de projeto.

\subsection{Parâmetros iniciais adotados}

Para a representação do tráfego, foram adotados quatro diferentes e crescentes valores de Número N, que variam de leve a muito pesado, conforme Tabela 2, e determinados conforme o The United States Army Corps of Engineers (USACE) e a American Association of State Highway and Transportation Officials (AASHTO).

Tabela 2: Valores de Número $\mathrm{N}$ adotados

\begin{tabular}{lll}
\hline Tráfego & USACE & AASHTO \\
\hline Leve & $1,00 \mathrm{E}+06$ & $7,00 \mathrm{E}+05$ \\
Moderado & $1,00 \mathrm{E}+07$ & $7,00 \mathrm{E}+06$ \\
Pesado & $1,00 \mathrm{E}+08$ & $7,00 \mathrm{E}+07$ \\
Muito Pesado & $1,00 \mathrm{E}+09$ & $7,00 \mathrm{E}+08$ \\
\hline
\end{tabular}

Para o subleito, foram adotados três diferentes e crescentes valores de capacidade de suporte (CBR), representando valores de capacidade de suporte que variam com a seguinte classificação: 4\% (ruim), $8 \%$ (regular) e $12 \%$ (bom).

No que diz respeito aos materiais das camadas superiores - base, sub-base e reforço do subleito foram utilizados Brita Graduada Simples (BGS), Brita Graduada Tratada com Cimento (BGTC) e Macadame, além do CAUQ para o revestimento.

Após o dimensionamento pelo método empírico do DNIT, as estruturas calculadas foram analisadas por intermédio dos procedimentos mecanicistas. Como descrito anteriormente, a análise mecanicista consiste no cálculo dos valores de tensões, deformações e deflexões que atuam nos pontos críticos das 
estruturas analisadas quando da passagem de um determinado Número N para, posteriormente, compará-los com os valores máximos admissíveis obtidos através de modelos de desempenho. Os cálculos dos valores atuantes são realizados com o auxílio de softwares, sendo, neste trabalho, utilizado o ELSYM5 .

Os dados de entrada do programa ELSYM-5 são as espessuras de cada camada (em $\mathrm{cm}$ ) e os valores de Coeficiente de Poisson e Módulo de Elasticidade (não de MR, pois este é um intervalo de valores descrito por uma equação cujo resultado varia em função da carga, enquanto aquele é um valor fixo, geralmente adotado o valor mais desfavorável desse intervalo). São dados também os pontos de aplicação das cargas e seus respectivos valores (em kgf), e os pontos críticos da análise, localizados no plano tridimensional com coordenadas cartesianas. Na Tabela 3, verificam-se os principais parâmetros adotados no ELSYM-5.

Tabela 3: Valores de Módulo de elasticidade e Coeficiente de Poisson adotados

\begin{tabular}{|c|c|c|}
\hline Material & Módulo de Elasticidade $\left(\mathrm{kgf} / \mathrm{cm}^{2}\right)$ & Coeficiente de Poisson \\
\hline Concreto Asfáltico & Variável (Hipóteses) & 0,30 \\
\hline Brita Graduada Simples (BGS) & 3.000 & 0,35 \\
\hline Brita Graduada Tratada com Cimento (BGTC) & 70.000 & 0,25 \\
\hline Macadame & 2.000 & 0,40 \\
\hline Solo Natural de Subleito $(C B R=4 \%)$ & 400 & 0,45 \\
\hline Solo Natural de Subleito $(C B R=8 \%)$ & 800 & 0,45 \\
\hline Solo Natural de Subleito (CBR = 12\%) & 1.200 & 0,45 \\
\hline
\end{tabular}

A observação detalhada do tipo de estrutura dimensionada permite identificar quatro pontos críticos a serem analisados, conforme as expressões de (1) a (4). Ressalte-se que as equações de fadiga utilizadas para a análise foram adotadas neste trabalho por serem recomendadas por DNIT (2006), com o intuito de ser fidedigno ao procedimento normativo em vigor e avaliar suas condições de dimensionamento.

a) Deflexão vertical recuperável no topo do revestimento, conforme DNER (1994) e exposto na expressão (1):

$$
\mathrm{D}_{\mathrm{adm}}=10^{\left(3,148-0,188 \cdot \log \mathrm{N}_{\mathrm{USACE}}\right)}
$$

b) Deformação horizontal de tração na fibra inferior do revestimento, equação do Instituto de Asfalto recomendada por DER/SP (2006), e descrita na expressão (2):

$$
\varepsilon \mathrm{t}_{\mathrm{adm}}=2,2 \cdot 10^{-2} \cdot\left(\frac{1}{\mathrm{~N}_{\mathrm{AASHTO}}}\right)^{0,304}
$$

c) Tensão horizontal de tração na fibra inferior da BGTC, proposta por Balbo (2009), e apresentada na expressão (3);

$$
\mathrm{N}_{\mathrm{f}}=10^{(17,137-19,608 \cdot \mathrm{RT})}
$$

d) Deformação vertical de compressão no topo do subleito apresentando por Dormon e Metcalf (1965) apud DER/SP (2006), sendo expressa por (4).

$$
\varepsilon \mathrm{V}_{\mathrm{adm}}=\frac{1}{\left[\sqrt[4,762]{\left(\frac{\mathrm{N}_{\mathrm{USACE}}}{6,070 \cdot 10^{-10}}\right)}\right]}
$$

\section{ANÁLISE E DISCUSSÃO DOS RESULTADOS}

Na Figura 1 é possível conferir as estruturas resultantes do dimensionamento empírico para os respectivos Números $\mathrm{N}$ (especificados como leve, moderado, pesado e muito pesado), um período de projeto de 10 anos, considerando solos do subleito com CBR de 4\%, 8\% e 12\% e um MR constante.

Equacionar o comportamento do revestimento asfáltico de um pavimento implica também se identificar a intensidade que as diversas variáveis atuarão sobre a estrutura e como a resultante dessas variáveis descreverão o comportamento do MR do CAUQ ao longo de todo o seu período de projeto. 


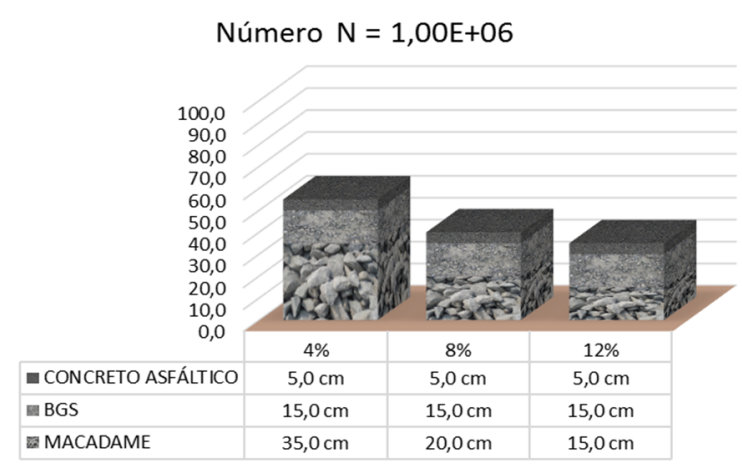

(a)

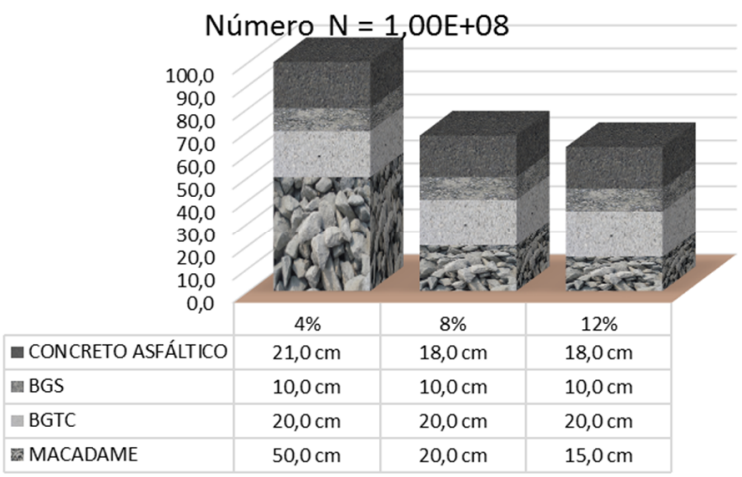

(c)

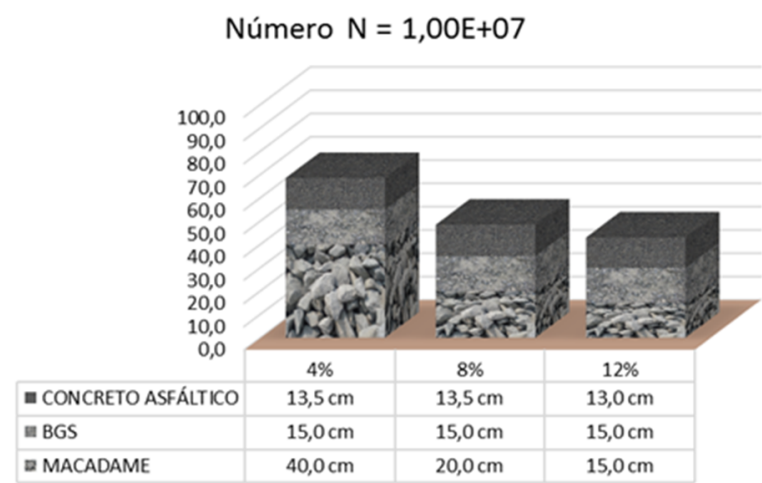

(b)

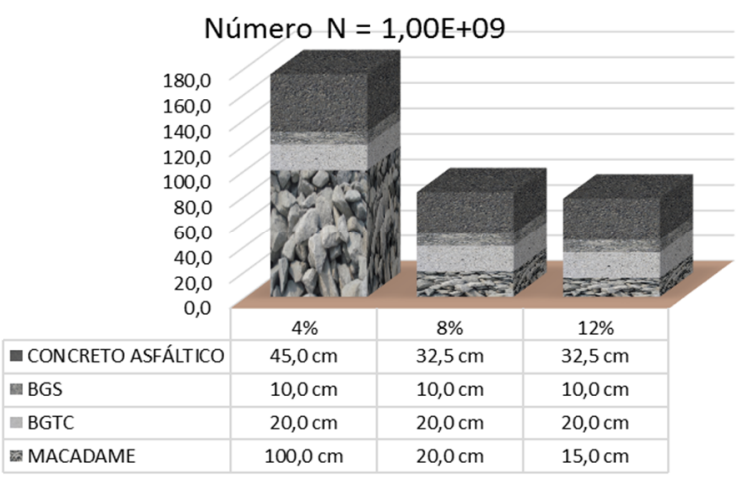

(d)

Figura 1. Estruturas dimensionadas pelo método empírico e verificadas considerando um MR constante e tráfego (a) Leve, (b) Moderado, (c) Pesado e (d) Muito Pesado

Assim, com o intuito de se realizar uma verificação teórica de quanto essas variações de MR podem interferir no período de projeto do pavimento e sabendo-se que essas variações podem resultar em MR maiores que o MR inicial (resultado do envelhecimento, por exemplo) ou menores (resultado do aumento significativo da temperatura, por exemplo), arbitrou-se pelas suposições de comportamento do MR dispostas na Figura 2. As adoções foram atribuídas para tendências de MR muito crescentes, crescentes, decrescentes e muito decrescentes.

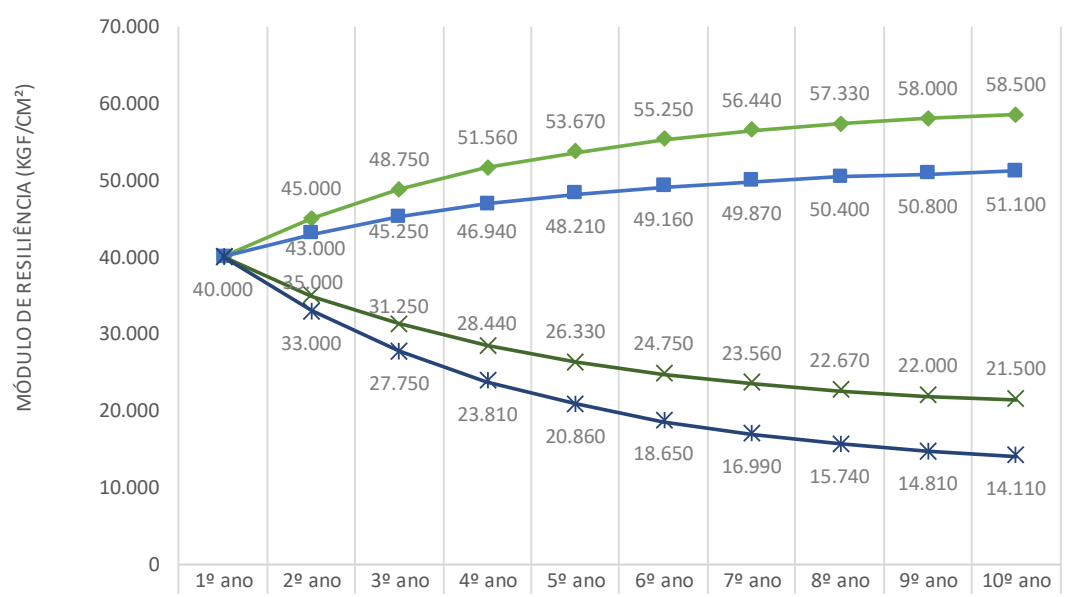

Figura 2. Hipóteses de variação do MR do CAUQ em função do período de projeto

Na Tabela 4 é possível observar as estruturas dos pavimentos dimensionadas pelo método empírico e as respectivas verificações mecanicistas. Verifica-se que, exceto para o pavimento submetido ao tráfego 
leve, em que todas as estruturas atendem aos parâmetros máximos admissíveis das equações de desempenho estudadas, todas as demais necessitam de ajustes e aumentos de espessuras.

Tabela 4: Estruturas dimensionadas e verificadas para diversos níveis de tráfegos

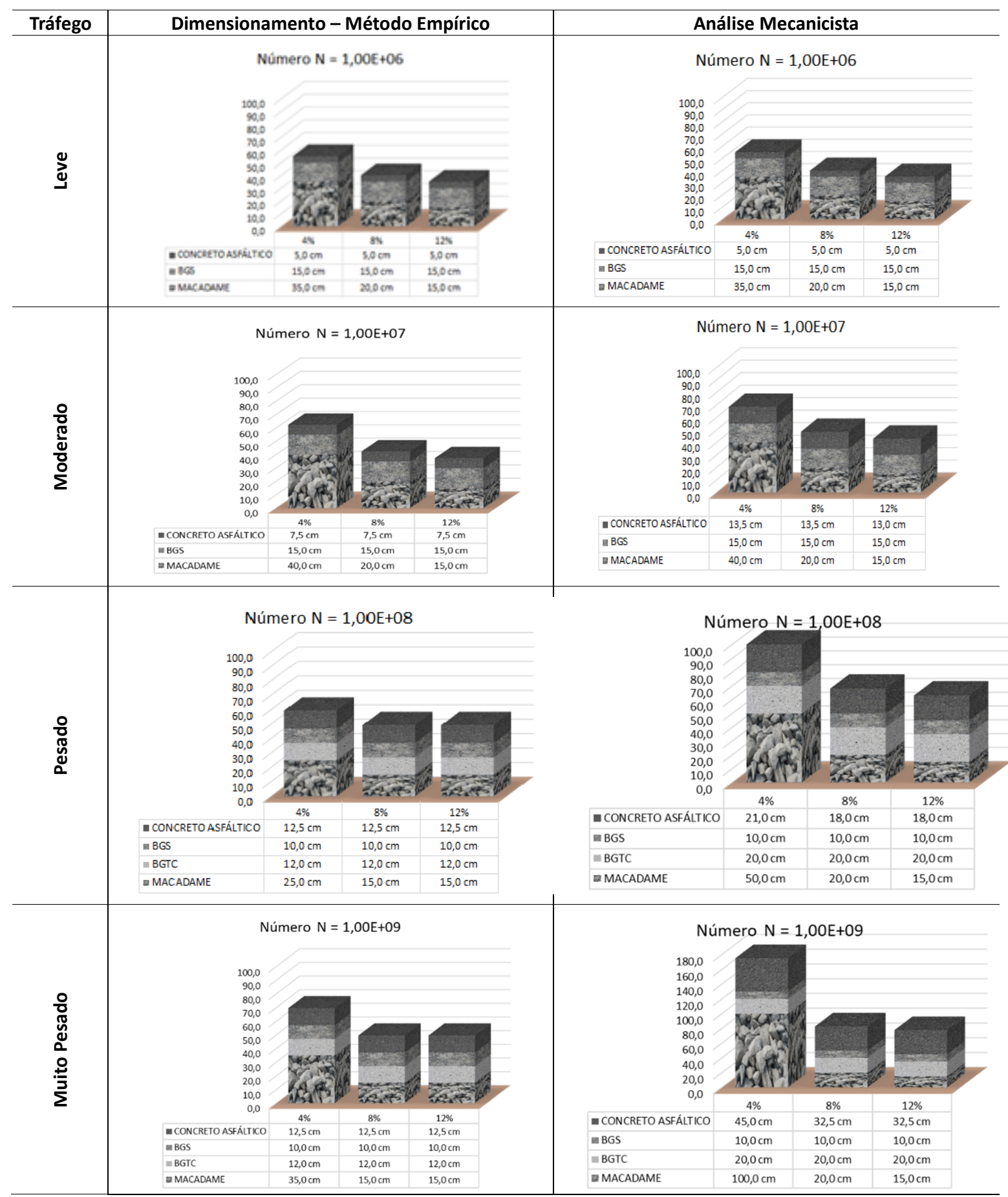

Arbitrados os diversos valores de MR, cada uma das 12 estruturas dimensionadas (4 categorias de Número $\mathrm{N}$ versus 3 condições distintas de CBR para o subleito) com MR constante foi submetida a mais

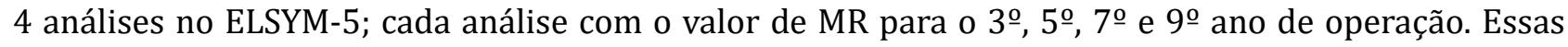
análises resultaram em valores de tensões, deformações e deflexões atuantes que alimentaram novamente os modelos de desempenho. Os resultados em um valor de Número $\mathrm{N}$ puderam ser traduzidos como a vida útil remanescente em anos. Essas análises foram repetidas, também, para cada hipótese de MR, resultando em 192 interações. 


\begin{tabular}{|c|c|c|c|c|c|c|c|c|}
\hline \multicolumn{3}{|c|}{ Ano da Análise: } & \multicolumn{3}{|c|}{ Início do 30 ano } & \multicolumn{3}{|c|}{ Início do 70 ano } \\
\hline Pavimento: & \multicolumn{2}{|r|}{ P4MD } & \multicolumn{3}{|c|}{ |Vida de Projeto } & \multicolumn{3}{|c|}{ Vida de Projeto } \\
\hline Tráfego: & \multirow{6}{*}{$\begin{array}{r}120,0 \\
100,0 \\
80,0 \\
60,0 \\
40,0 \\
20,0 \\
0,0\end{array}$} & & \multirow{2}{*}{11,7} & & & 0,0 & & \\
\hline Pesado & & & & & & 3,3 & & \\
\hline CBRp: & & 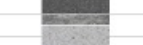 & 16,8 & & & 0,0 & & \\
\hline $4 \%$ & & wasts & & & & & & \\
\hline Hipótese de Módulo: & & कीज & \multirow{3}{*}{\multicolumn{3}{|c|}{ 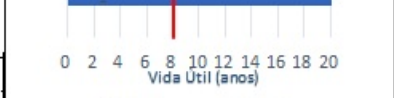 }} & \multirow{3}{*}{\multicolumn{3}{|c|}{ 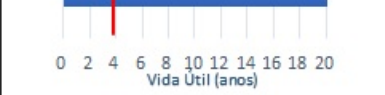 }} \\
\hline Muito Decrescente & & & & & & & & \\
\hline Revestimento (cm): & & $\begin{array}{l}21,0 \\
10,0\end{array}$ & & & & & & \\
\hline Base $(\mathrm{cm})$ : & & $\begin{array}{l}10,0 \\
20,0\end{array}$ & \multirow{2}{*}{\multicolumn{3}{|c|}{$\begin{array}{l}\text { - Deflexão } \quad \text { Def. CA } \\
\text { " Tensão BGTC }=\text { Def. Sub. }\end{array}$}} & \multirow{2}{*}{\multicolumn{3}{|c|}{$\begin{array}{l}\text { =Deflexão }=\text { Def. CA } \\
\text { =Tensão BGTC } \text { —Def. Sub. }\end{array}$}} \\
\hline Sub-Base $(\mathrm{cm})$ : & & 20,0 & & & & & & \\
\hline Reforço $(\mathrm{cm})$ : & & 50,0 & & & & & & \\
\hline Vida Remane & cente de & Projeto: & & 8 anos & & & 4 anos & \\
\hline Númerc & N (USAC & & & $8,23 E+07$ & & & $4,36 E+07$ & \\
\hline Número & $V$ (AASHT & & & $5,76 E+07$ & & & $3,05 E+07$ & \\
\hline Módulo d & Elasticid & ade: & & 27.750 & & & 16.990 & \\
\hline & & & Atuante & N remanescente & Vida Útil & Atuante 1 & N remanescente & Vida Útil \\
\hline Deflexão no Topo do Re & $-D_{0}(1 / 1$ & $00 \mathrm{~mm}$ ): & 46,0 & $7,91 \mathrm{E}+07$ & 5,7 & 49,1 & $5,60 E+07$ & 0,0 \\
\hline Deformação de Tração n & $C A-\varepsilon t_{h}$ & $\mathrm{~cm} / \mathrm{cm}):$ & $8,67 \mathrm{E}-05$ & $9,83 E+07$ & 11,7 & $1,09 \mathrm{E}-04$ & $7,07 E+07$ & 3,3 \\
\hline Tensão de Tração no BGT & $-\sigma t_{h}(k g$ & $\left.\mathrm{f} / \mathrm{cm}^{2}\right):$ & 3,16 & $1,93 E+08$ & 16,8 & 3,39 & $4,38 E+07$ & 0,0 \\
\hline Def. Vertical no Topo do & ubleito & $\varepsilon v(\mathrm{~cm} / \mathrm{cm}):$ & 7,41E-05 & $2,83 \mathrm{E}+10$ & 2747,1 & $7,96 \mathrm{E}-05$ & $2,00 E+10$ & 1834,2 \\
\hline Anod & Análise & & & Início do 3o ano & & & Início do 90 ano & \\
\hline Pavimento: & & P8MD & & |Vidade Pro & ojeto & & Vida de Projeto & \\
\hline Tráfego: & & & 48,7 & 3,7 & & & 6,9 & \\
\hline Pesado & $\begin{array}{l}80,0 \\
600\end{array}$ & & 5,4 & & & op & p & \\
\hline CBRp: & $\begin{array}{l}60,0 \\
40,0\end{array}$ & & 1,7 & & & op & p & \\
\hline $8 \%$ & & & & & & & & \\
\hline Hipótese de Módulo: & & 2ants & & & & & | & \\
\hline Muito Decrescente & & & & 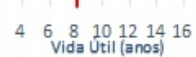 & 61820 & & & \\
\hline Revestimento (cm): & & 18,0 & & Vida Útil (anos) & & & Vida Ütil( (anos) & 20 \\
\hline Base $(\mathrm{cm})$ : & & 10,0 & & Deflexão $\quad$ Def. $\mathrm{CA}$ & & & $\begin{array}{l}=\text { Deflexão } \\
\text { Def. CA }\end{array}$ & \\
\hline Sub-Base $(\mathrm{cm})$ : & & 20,0 & & Tensão BGTC = Def. Su & & & & \\
\hline Reforço $(\mathrm{cm})$ : & & 20,0 & & & & & & \\
\hline Vida Remane & cente de & Projeto: & & 8 anos & & & 2 anos & \\
\hline Númerc & N UUSAC & & & $8,23 E+07$ & & & $2,24 E+07$ & \\
\hline Número & $\checkmark$ (AASH & & & $5,76 E+07$ & & & $1,57 E+07$ & \\
\hline Módulo d & Elasticic & lade: & & 27.750 & & & 14.810 & \\
\hline & & & Atuante & N remanescente & Vida Útil & Atuante & e N remanescente & Vida Útil \\
\hline Deflexão no Topo do Re & $-D_{0}(1)$ & $00 \mathrm{~mm}$ ): & 32,3 & $5,21 E+08$ & 48,7 & 36,3 & $2,79 E+08$ & 16,9 \\
\hline Deformação de Tração n & $C A-\varepsilon t_{h}$ & $(\mathrm{~cm} / \mathrm{cm}):$ & $1,04 \mathrm{E}-04$ & $5,34 E+07$ & 5,4 & $1,38 E-04$ & $3,63 E+07$ & 0,0 \\
\hline Tensão de Tração no BG & $c-\sigma_{\mathrm{h}}(\mathrm{k}$ & $\left.\mathrm{f} / \mathrm{cm}^{2}\right):$ & 3,41 & $3,85 E+07$ & 1,7 & 3,75 & $4,29 E+06$ & 0,0 \\
\hline Def. Vertical no Topo do & Subleito & $\varepsilon v(\mathrm{~cm} / \mathrm{cm}):$ & $1,04 \mathrm{E}-04$ & $5,55 \mathrm{E}+09$ & 537,1 & $1,13 \mathrm{E}-04$ & $3,79 E+09$ & 329,6 \\
\hline Anod & Análise: & & & Início do 30 ano & & & Início do 50 ano & \\
\hline Pavimento: & & P12MD & & Vida de Proj & jeto & & Vida de Projetc & \\
\hline Tráfego: & & & 181,1 & & & 127, & 7,9 & \\
\hline Pesado & & & 5,2 & & & 1,7 & & \\
\hline CBRp: & $\begin{array}{l}60,0 \\
40,0\end{array}$ & $\sin ^{2}$ & 13,5 & & & 1,7 & & \\
\hline $12 \%$ & & $\therefore$ & & & & & & \\
\hline Hipótese de Módulo: & & zescest & & \begin{tabular}{l|l|}
1 & $\mid$
\end{tabular} & & I & $|1|+\mid$ & \\
\hline Muito Decrescente & & & & $4 \quad 6 \quad 8101214161$ & 1820 & & 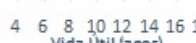 & 1820 \\
\hline Revestimento $(\mathrm{cm})$ : & & 18,0 & & Vidà Útil (anos) & & & Vida Útil (anos) & \\
\hline Base $(\mathrm{cm})$ : & & 10,0 & & Deflerão Def. CA & & & Deflexão $=$ Def. CA & \\
\hline Sub-Base $(\mathrm{cm})$ : & & 20,0 & & 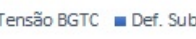 & & & Tensão BGTC $\backsim$ Def. Sub. & \\
\hline Reforço (cm): & & 15,0 & & & & & & \\
\hline Vida Remanes & rente de & Projeto: & & 8 anos & & & 6 anos & \\
\hline Número & N USAC & & & $8,23 E+07$ & & & $6,35 E+07$ & \\
\hline Número & I (AASHT & & & $5,76 E+07$ & & & $4,45 E+07$ & \\
\hline Módulo de & Elasticid & ade: & & 27.750 & & & 20.860 & \\
\hline & & & Atuante & N remanescente & Vida Útil & Atuante & N remanescente & Vida Útil \\
\hline Deflexão no Topo do Rev & $-D_{0}(1 / 1$ & $00 \mathrm{~mm}$ ): & 25,4 & $1,88 E+09$ & 181,1 & 26,8 & $1,40 E+09$ & 127,9 \\
\hline Deformação de Tração nc & $C A-\varepsilon t_{h} !$ & $\mathrm{cm} / \mathrm{cm}):$ & $1,05 E-04$ & $5,18 E+07$ & 5,2 & $1,21 E-04$ & $4,23 E+07$ & 1,7 \\
\hline Tensão de Tração no BGT & $-\sigma t_{h}(k g$ & $\left.\mathrm{f} / \mathrm{cm}^{2}\right):$ & 3,19 & $1,59 E+08$ & 13,5 & 3,34 & $6,04 E+07$ & 1,7 \\
\hline Def. Vertical no Topo do & ubleito - & $\varepsilon v(\mathrm{~cm} / \mathrm{cm}):$ & $8,78 \mathrm{E}-05$ & $1,26 \mathrm{E}+10$ & 1221,3 & $9,12 \mathrm{E}-05$ & $1,05 E+10$ & 989,2 \\
\hline
\end{tabular}

Figure 3. Exemplos de análise da variação da vida útil em função da variação do módulo 
A Figura 3 exemplifica três dessas interações para alguns anos de análise referidos no parágrafo anterior. A estrutura foi dimensionada para um tráfego pesado (P), considerando as três condições de CBR (ruim, regular e bom, respectivamente, iguais a 4\%, 8\% e 12\%), na situação de hipótese de módulo muito decrescente (MD). Os pavimentos são identificados nas planilhas como P4MD, P8MD e P12MD.

Observa-se que, de maneira geral, os três pavimentos propostos e analisados não atendem satisfatoriamente à verificação mecanicista, apresentando vida útil remanescente inferior às analisadas (as células vermelhas indicam essa condição). Exceto pela deformação vertical no topo do subleito, todos os demais pontos críticos - deflexão no topo do revestimento, deformação de tração no CAUQ e tensão de tração na BGTC - apresentam condição não conforme para a estrutura do pavimento.

Para melhor análise dos resultados, foi realizada verificação mecanicista em todos os pavimentos dimensionados para todas as hipóteses de módulo. Assim, utilizaram-se os modelos de desempenho recomendados por DNIT (2006) para correlacionar o comportamento teórico dos materiais previsto no dimensionamento com o comportamento dos materiais em campo.

Diante do exposto, pode-se afirmar que o decréscimo acentuado do MR do CAUQ possibilita ao pavimento o surgimento precoce de patologias diversas, tais como trincamentos por fadiga ou deformações antes do término do período de projeto. A Tabela 6 apresenta um resumo em termos percentuais de todas as 192 interações analisadas neste trabalho.

Tabela 6: Resumo da Variação de Vida Útil

\begin{tabular}{llll}
\hline Tráfego & CBRsubleito & Hipóteses de MR & Variação do Período de Projeto \\
\hline Leve & Ruim, Regular e Bom & Todas & Sem variação significativa \\
Moderado, Pesado e Muito Pesado & Ruim, Regular e Bom & Decrescente e Muito Decrescente & Perda de 14\% a 78\% \\
Moderado, Pesado e Muito Pesado & Ruim, Regular e Bom & Crescente e Muito Crescente & Ganho de 23\% a 172\% \\
\hline
\end{tabular}

\section{CONCLUSÕES}

Nas análises efetuadas neste trabalho, foi possível concluir que o Módulo de Resiliência (MR) do Concreto Asfáltico Usinado à Quente (CAUQ) tem interferência significativa nos projetos de dimensionamento de pavimentos rodoviários. Percebeu-se que utilizá-lo de modo constante ao longo de toda a operação do pavimento pode não proporcionar um desempenho satisfatório para a estrutura projetada, bem como não garantir a vida útil prevista.

Nas estruturas dimensionadas e analisadas neste trabalho, sobretudo quando submetidas à verificação mecanicista, em todos os pavimentos dimensionados para todas as hipóteses de módulo, foi possível entender que questões relacionadas ao tráfego, à usinagem, às espessuras das camadas, à temperatura e à radiação solar promovem variações maiores ou menores do MR do CAUQ. Tais situações permitem o desenvolvimento de defeitos relacionados à oxidação ou enrijecimento do revestimento, o que, por consequência, reduz a sua vida útil. Nesse sentido, recomenda-se que os efeitos ambientais sobre o revestimento sejam considerados pelos métodos de dimensionamento mecanístico, inclusive o que vem sendo desenvolvido no Brasil.

De acordo com a teoria de dimensionamento, se o CAUQ apresentar um MR muito crescente, devido à combinação entre clima, tipo de ligante, modificador, envelhecimento e material granulométrico, isso poderá acrescentar substancialmente a vida útil do pavimento. Para pesquisas futuras, sugere-se avaliar, também, o comportamento da fadiga do material em função do seu envelhecimento, haja vista as possíveis alterações da curva de fadiga inicial.

A partir do procedimento de cálculo previsto nas especificações vigentes, foi possível analisar de forma teórica, que pavimentos asfálticos quando dimensionados para atender um tráfego considerado moderado, pesado ou muito pesado, independente da capacidade de suporte do subleito, podem perder, já a partir do terceiro ano de operação, entre 14\% e 78\% da vida útil se o MR do CAUQ obedecer a uma 
tendência decrescente ou muito decrescente. Por sua vez, quando o MR do CAUQ obedecer a uma tendência crescente ou muito crescente, a vida útil do pavimento pode receber um acréscimo na ordem de $23 \%$ a $172 \%$.

Desse modo, espera-se que este trabalho contribua para o desenvolvimento de modelos de desempenho de pavimentos que considerem os principais fatores que interferem no comportamento do MR do CAUQ ao longo do período de projeto, de modo que se elaborem metodologias mais efetivas e racionais.

\section{REFERÊNCIAS BIBLIOGRÁFICAS}

Balbo, J. T. (2007) Pavimentação asfáltica: materiais, projetos e restauração. São Paulo: Oficina de Textos.

Balbo, J. T. (2009) Pavimentos de concreto. São Paulo: Oficina de Textos.

Beer, F. P. e Johnston Junior, E. R. (1995) Resistência dos materiais. 3. ed. São Paulo: Makron Books.

Bernucci, L. B., Motta, L. M. G., Ceratti, J. A. C e Soares, J. B. (2008) Pavimentação asfáltica: formação básica para engenheiros. Rio de Janeiro: Petrobrás; Abeda.

Departamento de Estradas de Rodagem do Estado de São Paulo (2006) Projeto de Pavimentação. DER. IP-DE-P00/001. São Paulo.

Departamento Nacional de Infraestrutura de Transportes (1994) Solos - determinação do Índice de Suporte Califórnia utilizando amostras não trabalhadas - método de ensaio. ME 049. DNIT. Rio de Janeiro: IPR.

Departamento Nacional de Infraestrutura de Transportes (2006). Manual de pavimentação. 3. ed. DNIT. Rio de Janeiro: IPR

Magalhães, S. T. (2004) Misturas Asfálticas de Módulo Elevado para Pavimentos de Alto Desempenho. Dissertação (Mestrado) COPPE - UFRJ, Rio de Janeiro.

Rohde, L. (2007) Estudo de Misturas Asfálticas de Módulo Elevado para Camadas Estruturais de Pavimentos. Tese (Doutorado) UFRGS - Rio Grande do Sul.

Senço, W. (1997) Manual de Técnicas de Pavimentação. São Paulo: Pini.

Severi, A. A., Balbo, J. T. e Rodolfo, M. P. (1998) Conceitos mecanicistas básicos sobre pavimentos asfálticos. São Paulo: Universidade de São Paulo - USP. 